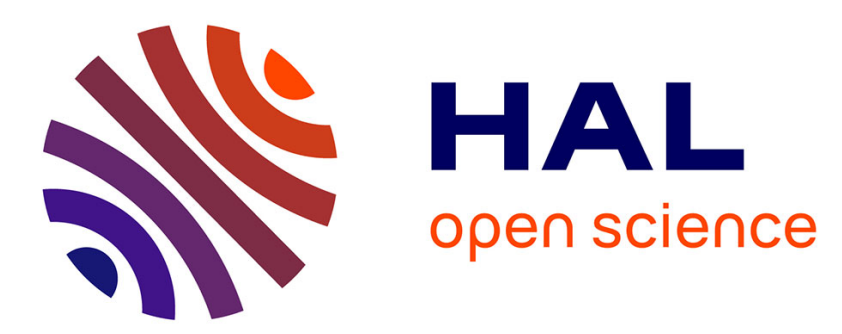

\title{
A Robust Scheme for Aggregating Quasi-Blind Robots in an Active Environment
}

Nazim Fatès, Nikolaos Vlassopoulos

\section{To cite this version:}

Nazim Fatès, Nikolaos Vlassopoulos. A Robust Scheme for Aggregating Quasi-Blind Robots in an Active Environment. International Journal of Swarm Intelligence Research, 2012, 3 (3), pp.15. 10.4018/jsir.2012070105 . hal-00740630

\section{HAL Id: hal-00740630 https://hal.inria.fr/hal-00740630}

Submitted on 8 Apr 2021

HAL is a multi-disciplinary open access archive for the deposit and dissemination of scientific research documents, whether they are published or not. The documents may come from teaching and research institutions in France or abroad, or from public or private research centers.
L'archive ouverte pluridisciplinaire HAL, est destinée au dépôt et à la diffusion de documents scientifiques de niveau recherche, publiés ou non, émanant des établissements d'enseignement et de recherche français ou étrangers, des laboratoires publics ou privés. 
This text is a preprint provided for information purposes Please refer to the final version of this article: International Journal of Swarm Intelligence Research, IGI Global, 2012,3 (3), pp.66-80

\title{
A Robust Scheme for Aggregating Quasi- blind Robots in an Active Environment
}

\author{
Nazim Fatès and Nikolaos Vlassopoulos \\ INRIA Nancy - Grand Est, LORIA, Nancy, France
}

\begin{abstract}
The question of how to aggregate autonomous agents with limited abilities in the absence of centralized control or coordination is known as the Decentralized Gathering Problem. We present a bio-inspired aggregation scheme that solves this problem and study a first application of this scheme to a small team of robots. The robots (Alice and Khepera III) obey simple rules and have only a rudimentary perception of their environment. The collective behavior is based on stigmergic principles and uses an active environment to relay the communications between robots. This results in an aggregation process that shows good properties of robustness and that can in principle be extended to swarms of robots.
\end{abstract}

Keywords: Decentralized Gathering, Agent Aggregation, Swarm Robotics, Reaction-diffusionchemotaxis

\section{INTRODUCTION}

Let us consider a system consisting of a great number of autonomous robots randomly spread on a plane area, the environment. The robots need to gather to realize a given task, for instance to exchange information about the area or to collaborate to remove an obstacle. In the case where the agents are not aware of their position, orientation nor of the presence of other agents in their neighborhood and if furthermore there are no means of centralized coordination or communication, the question of their aggregation is known as the Decentralized Gathering Problem.

This problem has been studied before, using different assumptions about the agents and the environment. In an early work, Sugihara \& Suzuki (1996) deal with the distributed coordination of a swarm of robots in order to form geometrical shapes. The work presented by Garnier et al. $(2008$; 2009) studies the emergent aggregation of robots that mimic the behavior of Blattela germanica, while Ando et al. (1999) and Flocchini et al. (2005) study the aggregation problem 
under the assumption of limited visibility. A theoretical study by Prencipe (2007) explored the complexity of the problem of decentralized gathering with simplifying hypotheses.

One approach to solving the decentralized gathering problem consists of imitating the social amoeba species Dictyostelium discoideum (Fatès, 2010). The global dynamics of this aggregation scheme were first studied in the scope of cellular automata (CA) modeling. However, this scheme is more generic as it can be considered as a high-level behavioral description. The key idea is the existence of an active environment that conveys simple messages among the agents, the virtual amoebae. The agents interact with the environment by either initiating the transmission of a message or by detecting the existence of messages in their local neighbourhood. These two ingredients form the basis for a stigmergic behavior.

The first advantage of this scheme is that both the agent description and the active environment are simple and straightforward to implement, at least in a simulation environment. The main idea behind the amoebae scheme is that the use of an active environment delegates to this environment parts of the complexity that would otherwise be implemented in the agents.

In previous simulations, the aggregation scheme was shown to be robust to different perturbations of the agents and the environment, e.g., to the presence of obstacles and noise in the movement of the agents. The first aim of this paper is to investigate how to transpose the aggregation scheme in the case of robotic agents. The second objective is to examine whether the robustness of the aggregation process is conserved with robots. We examine two different series of experiments that use physically different implementations of the same active environment. The first approach we will present is based on the ALICE micro-robots ${ }^{1}$. These robots feature a compact design but have a restricted set of abilities: the robots we had at our disposal were equipped with only two sensors that could measure the light intensity of a source located above the robots. The second approach uses a modified version of the Khepera III robots, in conjunction with an "interactive table" (ROMEA) that has been specifically developed for conducting robotic experiments (Beaufort et al., 2011). Although these robots have superior abilities, we made a minimalistic use of their features: our goal is indeed to present a simple method for aggregating the robots, in particular we want the experiment to be set rapidly without having to tune and calibrate too many parameters.

This paper is organized as follows: Section 2 presents the aggregation scheme while Section 3 describes the implementation on the two different types of robots. Following, Section 4 presents the advantages and drawbacks that we observed during our experiments, as well as the differences we observed between the two types of robots and environment implementations. Finally, we discuss the results in Section 5.

\section{THE VIRTUAL AMOEBAE AGGREGATION SCHEME}

\section{From Biology to Computer Science}

The virtual amoebae aggregation scheme draws its inspiration from the behavior of the social amoebae species Dictyostelium discoideum. During its life-cycle, this species undergoes a transformation from a mono-cellular to a multi-cellular organism. This transformation occurs in different phases. Initially, and when the environment is rich in nutrients, the individuals are scattered. When the concentration of nutrients decreases, the amoebae start to aggregate so as to

1 Designed at EPFL, Lausanne, Switzerland, 1998, see: http://en.wikipedia.org/wiki/Alice mobile robot 
form a single body, which moves until it finds a suitable environment. Once such an environment is found, the organism transforms again, forming a fruiting body. The fruiting body releases spores in the environment, and the cycle starts again.

The aggregation phase of $D$. discoideum is based on chemotaxis, that is, the ability to move preferentially towards a source that emits chemical products. In nature, the production of the chemical agent (cyclic AMP) within each amoeba is strated when the nutrients in the environment are lacking. The cAMP is secreted internally by the amoebae, and then emitted, which causes neighboring individuals to get closer to the emitting cell by following the gradient of the concentration of cAMP. This behavior amplifies until it results in the formation of small clusters that eventually aggregate to form a single body.

Our amoebae aggregation scheme is based on a similar concept. At each discrete time step, an agent may emit a "chemical message" with a given probability. The message is then transmitted through the environment detected by other agents, which, in reaction, move towards the source of the message. Compared to the natural phenomenon, the amoebae aggregation scheme uses simplified hypotheses. In order to reduce the complexity of the agents, the agents do not follow the gradient of the transmitted messages, but rather simply detect the wavefronts (the reason for such a choice will be explained later). This allows for the agents to work in an "all-ornothing" fashion, which, in turn, simplifies the requirements for agent sensors, so that they need minimal calibration. Further, to simplify the design of the environment the "messages" do not use "classical" diffusion as their transport mechanism. Instead, the aggregation scheme is based on the existence of an active environment that allows the waves to travel at a constant speed and without any attenuation or modification. This allows us to simplify the agent-environment interaction, since an agent can initiate a "message" wave in the environment by making a local modification in the environment.

\section{The Greenberg-Hastings Cellular Automaton}

The dynamics of the environment we employ emulates the Greenberg-Hastings cellular automaton (GHCA). This model emulates reaction-diffusion processes and has the following advantages: (a) it is easy to implement and simulate in software, (b) the reaction-diffusion wavefronts travel over arbitrary large distances in the environment without any attenuation, (c) wavefronts skirt round obstacles.

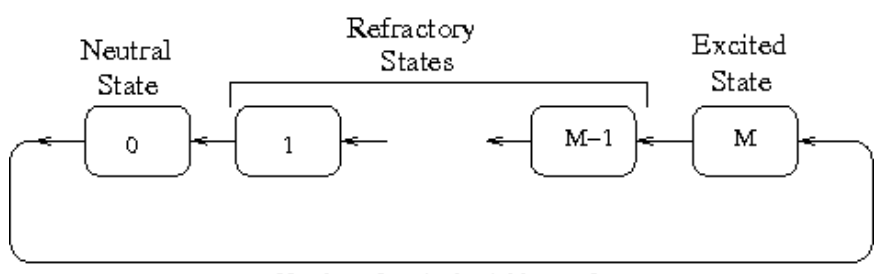

Number of excited neighbors $>0$

Figure 1: Cell rules as a state transition diagram

Formally, the discrete space is modeled with $\Lambda=\{1 \ldots L\} \times\{1 \ldots L\}$ as a square two-dimensional array of cells where $L$ determines the environment size. Each cell $c=\left(c_{x}, c_{y}\right)$ of $\Lambda$ is associated with a state in the set $\{0, \ldots, M\}$. We call 0 the neutral state, the states $M-1, \ldots, 1$ the refractory states, and $M$ the excited state. The state of a cell at time $t$ is denoted by $\sigma_{c}^{t}$. The state of each 
cell is updated in discrete times according to a function of its current state and the states of the cells in its neighborhood. If we denote by $E_{c}^{t}$ the fact that the neighborhood of a cell $c$ at time $t$ contains an excited state, then the state evolution of a cell, $c$ is described by the following set of equations (Fig. 1):

$$
\sigma_{c}^{t+1}=\left\{\begin{array}{c}
M, \quad \text { if } \sigma_{c}^{t}=0 \wedge E_{c}^{t} \\
\sigma_{c}^{t}-1, \text { if } \sigma_{c}^{t} \in M-1, \ldots, 1 \\
0, \quad \text { otherwise }
\end{array}\right\}
$$

In this work we consider three different types of neighborhoods: the 4-connected neighborhood, the 8-connected neighborhood, and a "circular neighborhood" that simulates the diffusion of isotropic waves. In all cases we assumed that the environment has free (or absorbing) boundary conditions; as a consequence, waves that reach the boundaries of the environment are absorbed.

In practice, this cellular automaton imitates the dynamics of reaction-diffusion processes: waves that start from excited cells expand outwards, so as to form wavefronts of excited cells followed by cells in refractory states (see Figure 2). When two wavefronts of excited cells collide, they annihilate.

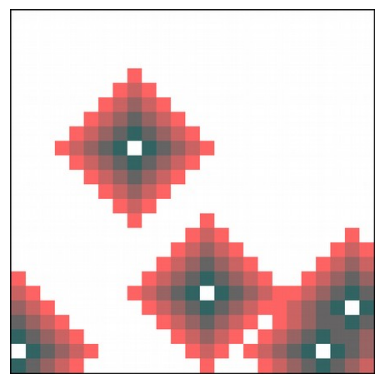

$\mathrm{t}=0$

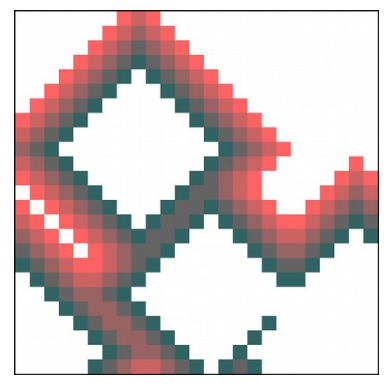

$t=5$

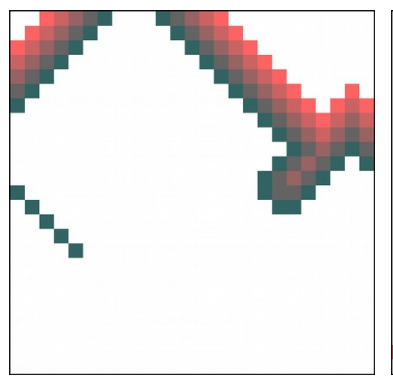

$t=10$

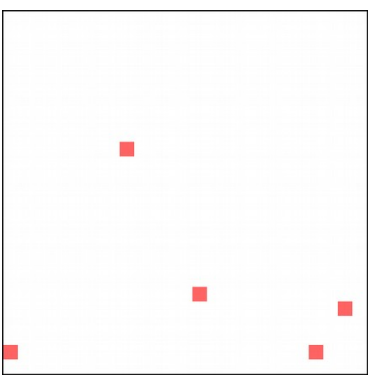

$t=15$

Figure 2: Reaction-diffusion process with a lattice size $\mathrm{L}=25, \mathrm{M}=4$ and5 initially excited cells. Excited cells are in bright red, refractory cells are in a red and grey mixture, neutral cells are in white.

\section{Coupling of the Virtual Amoebae with the Environment}

The basic model assumptions for the behavior of the virtual amoebae can be summarized as follows:

- Agents are autonomous and there is no direct communication among them.

- Each agent interacts only with its immediate environment (local interaction).

- Agents are not aware of their position, orientation or relative positions of other agents in the environment (quasi-blindness).

With the above in mind, the behavior of each agent can be described as follows:

1. If a reaction-diffusion wavefront is detected in the neighborhood of an agent, and the agent is not on a refractory cell then the agent moves towards the wavefront. 
2. If nothing is detected, then the agent may initiate a reaction-diffusion wave with a fixed probability $\lambda$, the firing rate.

\section{Characteristic Results from the CA Implementation of the Model}

Quantitative results regarding the aggregation characteristics have been presented in (Vlassopoulos \& Fatès, 2010). The most promising observations deal with the robustness of the aggregation scheme in the presence of obstacles in the environment as well as the the presence of perturbations in the movement of the agents. The ability of the virtual agents to aggregate in spite of various perturbations is mainly due to the properties of the GHCA. The reaction-diffusion wavefronts are capable of traveling around obstacles and regenerate when they travel through "holes" in obstacles. Figure 3 shows an example of aggregation in a rather constrained environment, consisting of rooms separated by doors.
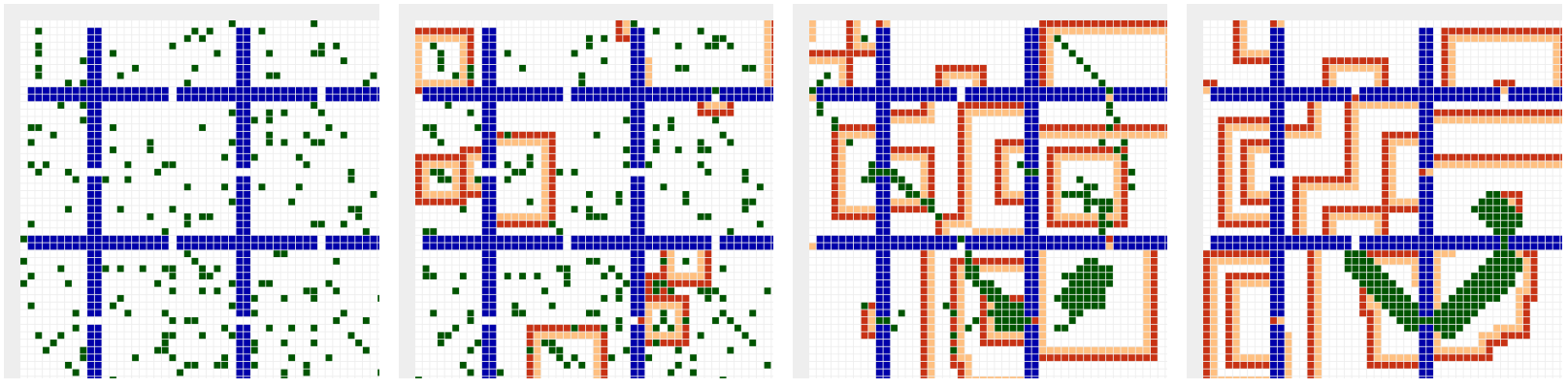

Figure 3: Agent aggregation in the presence of obstacles, for times $t=0,20,100$ and 700 . Agents are shown in green, obstacles in blue, neutral cells in white and excited cells with red.

To study the behavior with respect to perturbations in the agent movement, the original model introduced a "thermal motion" factor, so that each agent would jump to a neighboring cell with some probability $p_{a}$. Although this type of perturbation is quite different from what we would expect to observe in the application of the scheme to robot agents, still, it gives us a reason to believe that the model can be applied to robots. It is interesting to mention that the aggregation of the agents persisted even when the probability $p_{a}$ was quite high (up to 10\%). Figure 4 shows an example for $p_{a}=2 \%$. All these simulations results were rather encouraging; the challenge is now to see if this robustness can somehow be "transposed" in the case of robotic agents.
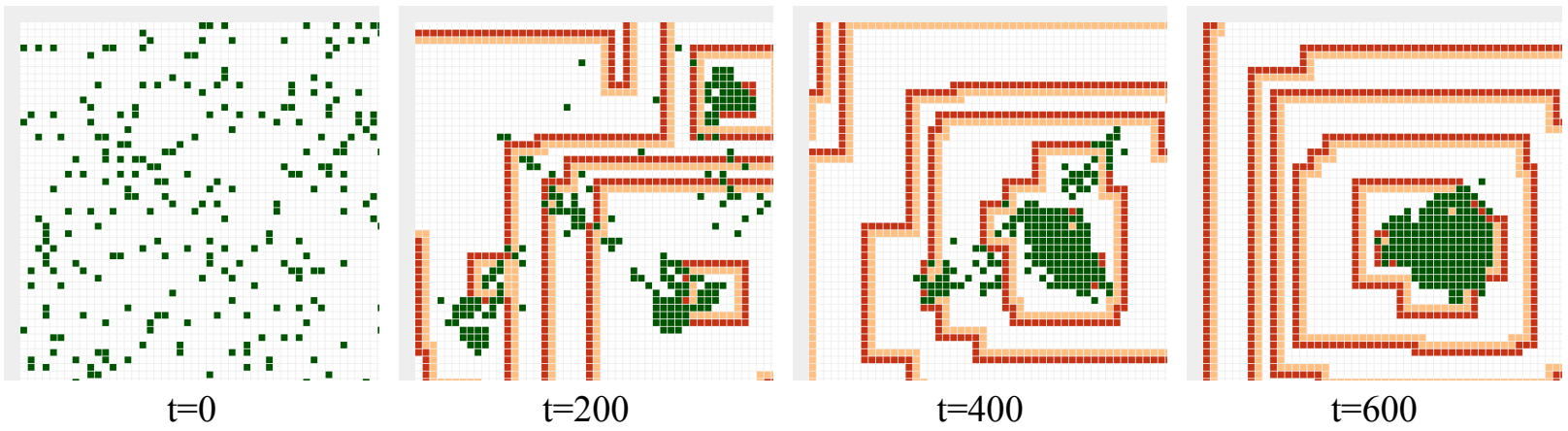
Figure 4: Example of the aggregation of the virtual amoebae in the presence of noise in the agent

movement

\section{FROM VIRTUAL AMOEBAE TO ROBOT AGENTS}

The main differences between the original CA-based instance of the aggregation scheme and its robot counterpart concern space and time. In simulations, both the agents and the environment operate in discrete time but in the physical experiments the movements of the robots are continuous. Moreover, in the discrete model, the actual size of the agents is neglected and each agent is simply assigned to a cell location. In the physical experiment, the agents have a nonnegligible size and they cover many environment cells. As a consequence, the relative length of the environment cells with respect to the agent dimensions become important, since they affect how the agents perceive and interact with the environment. The discretization time, that is, the time unit which separates two consecutive CA steps is also a key parameter. The other practical problem to solve is how to make the agents aware of the environment and how to enable them to interact with it. This section describes the solutions we studied for solving these problems.

\section{Chemotaxic Behavior}

Our starting point for designing the robots behavior was that we needed to adapt to the abilities of the ALICE robots (see Figure 5 for a picture). These robots are equipped with two wheels; their speed and direction is controlled by setting the speed of each wheel. Two light sensors are located on the top of the robot. These sensors are connected to analog-to-digital converters and are subject to high noise ratio.

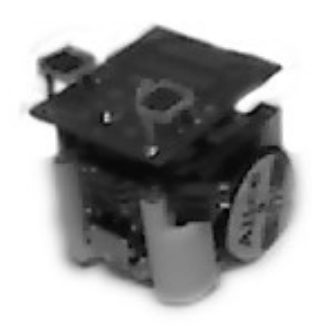

Figure 5: ALICE robot

With such robots, the main problem in applying the aggregation scheme is related to a problem of directional ambiguity: when detecting the waves with only two sensors, the robot can not distinguish between two incoming wavefronts with the same angle. This ambiguity is illustrated on Fig. 6: as the only available information is the different time of arrivals of the wavefronts, the agent can only determine if the wave is more or less parallel to the line that connects the two sensors. In other words, in the best case we are able to determine the absolute value of the angle of the incoming waves, but not its sign. 


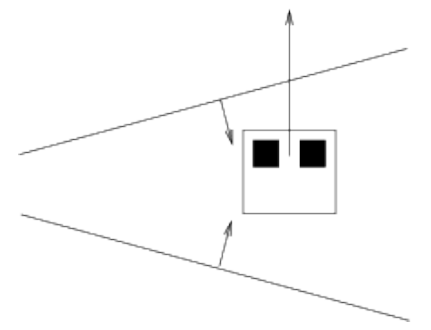

Figure 6: An example of the directional ambiguity, where the robot cannot distinguish between the two incident wavefronts

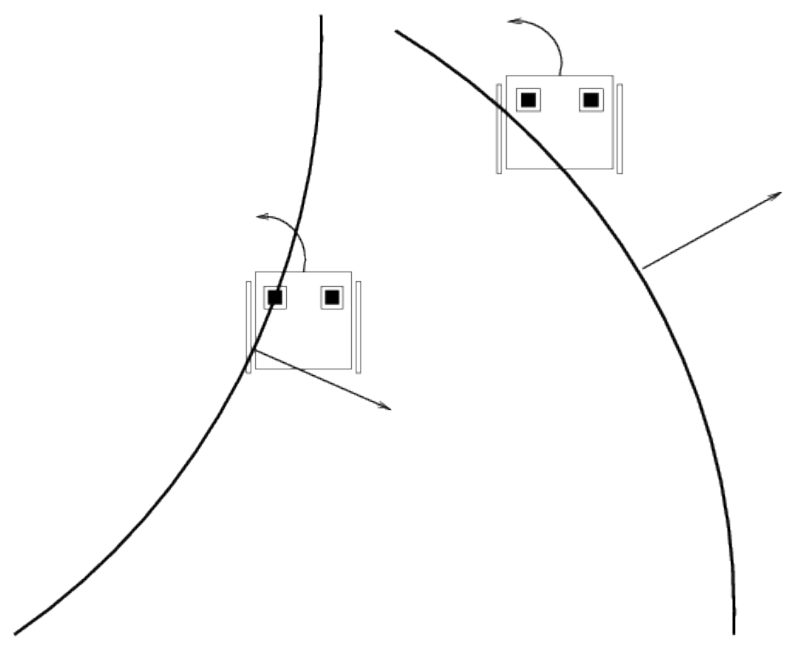

Figure 7: Elementary chemotaxis movements

The strategy we have chosen to overcome the directional ambiguity is to design a special movement for the robots. We programmed the robots to turn for an angle of $90^{\circ}$ towards the direction of the incident wave, that is, towards the first sensor that detects an incoming wave (Fig. 7). As a consequence, the robot will eventually turn towards the source of the waves, although this might require that more waves reach the robot. 
To understand why we did not want to follow the gradient of the incident reaction-diffusion waves, consdier the following case. Imagine that a reaction-diffusion wave, whose intensity gradually varies from a maximum value to zero is first detected by one of the sensors and after a moment from the other sensor. Assume that the agent eventually turns so that its direction is perpendicular to the incoming wavefronts, that is, it is now well positioned to go towards the

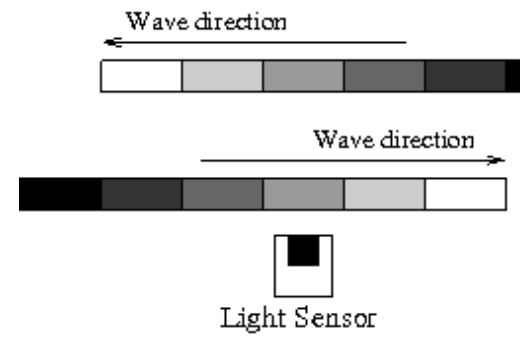

Figure 8: Example of the gradient ambiguity: the sensor will see the same temporal variations for a wave moving leftwards and a wave moving to the right

source. Since there are only two sensors and as they are placed perpendicular to the movement direction of the agent, they are now both parallel to the wavefronts. As a consequence, they become unable to accurately detect the gradient, since the light intensity varies in the same way on the two sensors, as it shown in Figure 8. This problem appears both when the robot faces towards or away from the reaction-diffusion waves. To avoid this second ambiguity, we programmed the robots to only react to the presence of wavefronts, that appear as a sudden change in the detected luminosity. When such a change is detected, the robot turns towards the sensor that detected the change. This rules allows a simple calibration and control logic for the sensors. More specifically, the decision on whether the robot should turn left or right is made only by calculating the difference of the measurements of the left and right sensor and comparing this difference to a threshold $T_{L}$.

The effect of these rules is that the agents follow a "zig-zag" track towards the source of the waves (see Fig. 9).

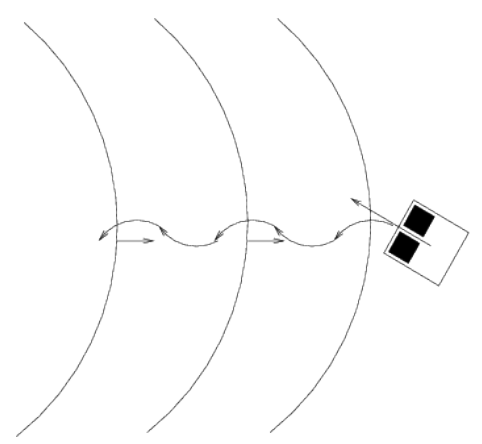

Figure 9: "Zig-zag" approach for moving towards the source of reaction-diffusion waves 


\section{Adapting the Agent Behavior}

One other problem with had to deal with was to ensure that the robots would not be affected by the reaction-diffusion waves that they initiated. This issue stems from the fact that the robot dimensions span several cells. An excitation that starts from a cell in the robot and extends outwards will eventually reach the sensors, and therefore the robot will "misunderstand" its own emission as an incident wave. Although we used a different approach to resolve this issue for the ALICE and KheperaIII robots, the most simple modification to the above model is to insert a refractory period after the initiation of a wave. During this refractory period the robot ignores all incoming waves.

The rules that govern each robot is shown in Fig. 10. Note that this figure also displays the agitation movements as well as the obstacle avoidance, which we will explain next.

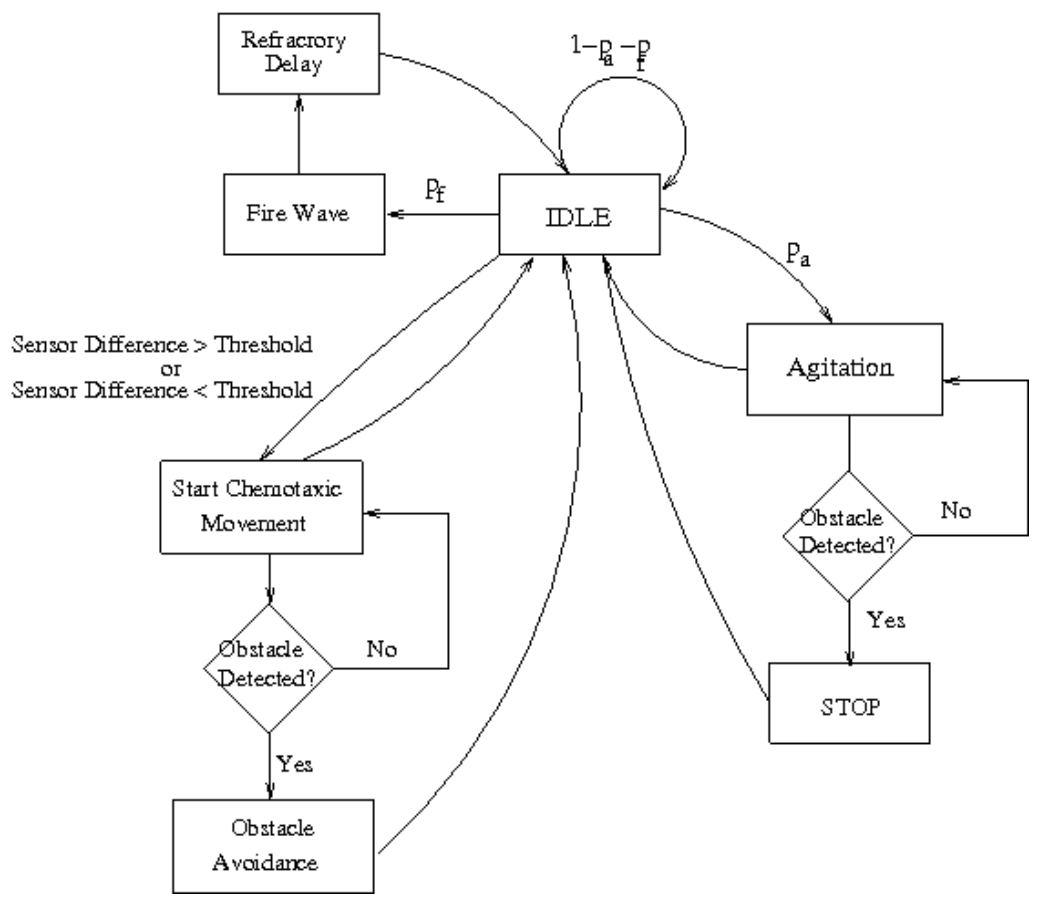

Figure 10: The complete state machine of the robot agents

The finite-state machine shown above covers the most complex cases that have been implemented in the KheperaIII robots. For the much simpler ALICE robots, where obstacle avoidance, agitatory moves and firing were not implemented on the robots, the state machine consisted of only 3 states, i.e. IDLE, MOVE LEFT and MOVE RIGHT.

\section{Experimental Setup for the environment}

As we have already seen, ALICE robots are equipped with two light sensors that are located on top of the robot. This arrangement was the basic guideline on the setup of the aggregation experiments, since the reaction-diffusion waves should be projected on the surface where the 
robots are located, from above. Following an idea by G. Theraulaz, we used a setup that was based on a camera and a projector: the images from the camera were processed in real-time in order to identify the positions of the robots in the environment and to map them to cell locations in the CA array. Knowing the agent locations and the state of the array, we simulated the evolution of the environment using FiatLux CA simulator ${ }^{2}$ and projected the resulting images back on the surface where the agents were located. Figure 11 shows a picture of one experiment, where we can see the agents and the projected waves.

\section{Interaction between robots and environment}

The main problem of this setup is that although the agents can "read" their environment and detect incoming wavefronts, it is not possible for them to "write" directly on the environment, that is,. to initiate a wave. In order to overcome this problem, we delegated the wave initiation part of the agent logic to the environment. However, since the fire decisions are not taken by the agents, it becomes impossible for them to know whether they should enter a refractory state and ignore their own emitted waves. The solution to this problem was again implemented in the environment, and more precisely by generating a "mask" that prevented from painting the first steps of the evolution of the reaction-diffusion process.

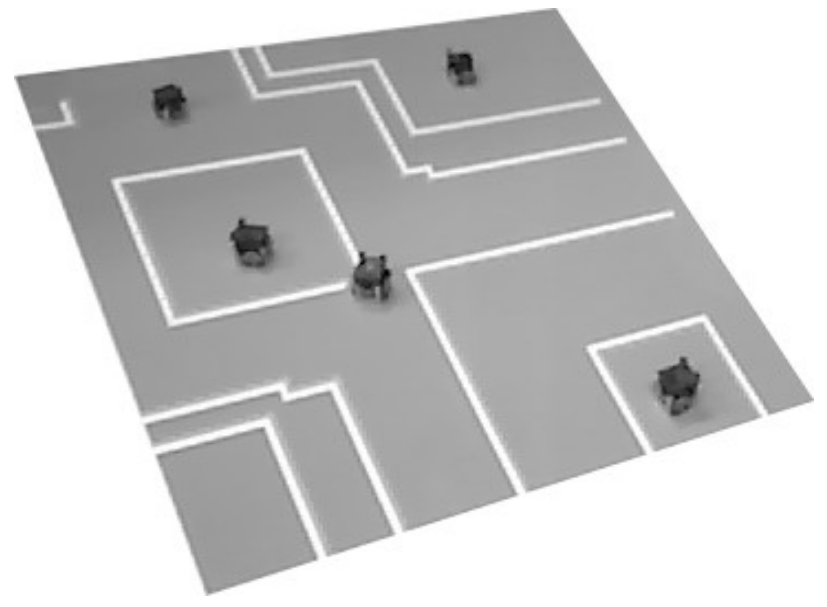

Figure 11: Snapshot of an experiment with ALICE robots, as taken from the camera that detects the robot positions.

In the case of the KheperaIII robots, setting up the experimental environment was greatly simplified, since the interactive ROMEA table and the existing programming interfaces provided all the tools required to track the position of the robots and simulate the reaction-diffusion process (see Fig. 12). The only drawback compared to the ALICE experiment was that we needed to work in total darkness because of a special tracking device that uses infrared leds that are situated below the KheperaIII robots.

2 http://fiatlux.loria.fr 


\section{Robot Parameters}

The final set of the parameters that characterize the model varied significantly in the two implementations. In the simplest case of the ALICE robots, the only things that the robot needed to know were the speed and movement duration for each wheel, as well as the value of the threshold, $T_{L}$. In the case of the KheperaIII robots, where the fire decisions were not merged with the environment logic, the parameter set included the fire probability, as well as the parameters for detecting other robots and the edges of the table.

This obstacle detection feature constitutes the major difference between the ALICE and KheperaIII experiments. Indeed, although the absence of obstacle avoidance in the first set of experiments resulted in the ALICE robots blocking and pushing each other, this behavior would only delay the aggregation. The robots would eventually unblock and succed to aggregate in a tight area. On the other hand, with the KheperaIII robots, using obstacle avoidance is mandatory since the robots should neither push each other, nor hit on the walls of the ROMEA table.

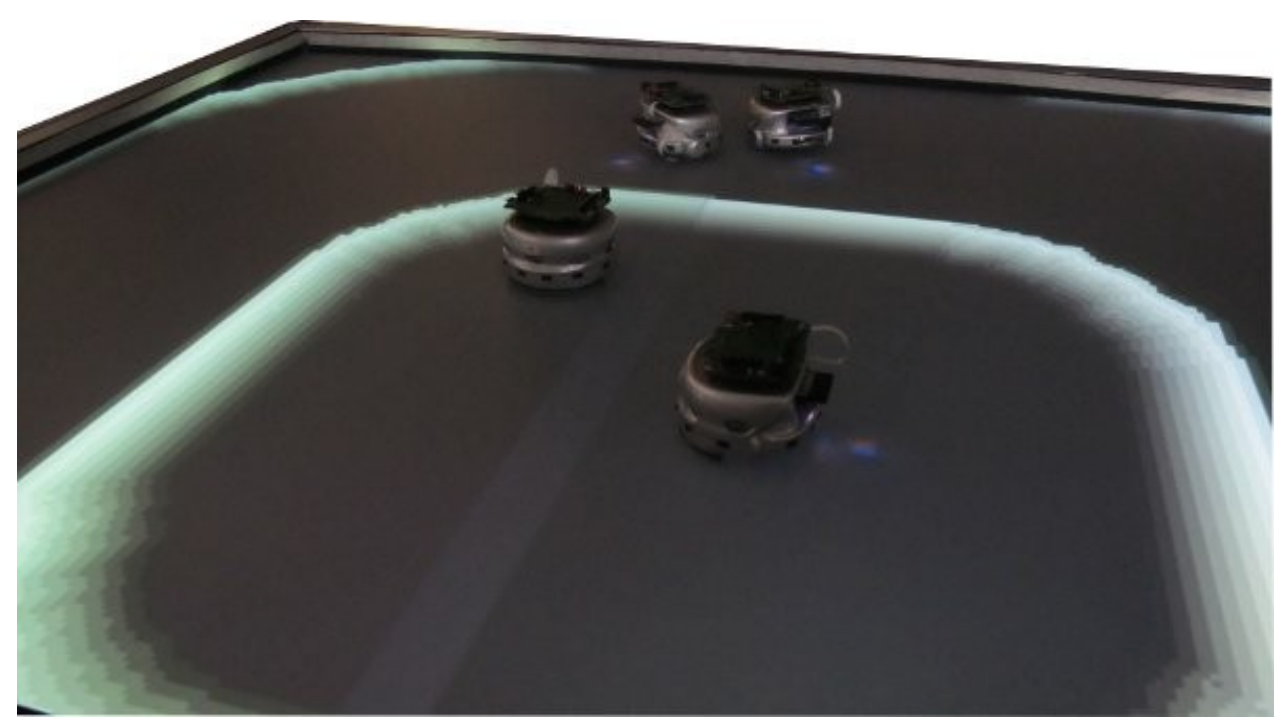

Figure 12: Snapshot of an experiment using the Khepera robots on the interactive ROMEA table. The experiment uses a "circular" neighborhood.

\section{A quick tuning of the parameters}

It is important to note that setting up the aggregation scheme for Alice or KheperaIII robots was done by tuning only a small number of parameters. More precisely, the main parameters that characterize the agents are the firing rate $\lambda$ and the refractory time, the turning angle and movement time constants. Regarding the environment, the main parameters are the number of states $M$ and the discretization time, that is, the time between two CA evolutions of the environment.

The tuning of these parameters were that the following balance was respected : 
1. In order to speed up the gathering process, both the robots and the environment should evolve as quickly as possible.

2. In order not to mix up informations sent by each robot, the dynamics of the environment, that is, the speed of the reaction-diffusion waves, should stay higher than the robots speed. This in particular guarantees that no wavefronts will be detected more than once.

3. The environment should not evolve too rapidly in order for the robots to have a sufficient time to detect the waves.

To tune the turning angle, we implemented a simplified model in software to emulate as closely as possible the ALICE robots, with configurable sensor and wheel positions as well as turning radius. Using this model we ran several simulations with various turning angles and also trying out cases where the angle was subject to noise. In all cases, the robots managed to converge, with the fastest average convergence time obtained for a fixed turning angle of $90^{\circ}$.

\section{EXPERIMENTS AND OBSERVATIONS}

Readers are encouraged to consult the AMYBIA project web-page ${ }^{3}$, which displays videos of our ALICE and KheperaIII experiments. Some experiments are also accessible through an applet that replays the recorded robot positions and orientation.

\section{Clear environment with Alice and KheperallI Robots}

Our first set of experiments was carried out with ALICE robots in an environment without obstacles. In such conditions, we could observe that the aggregation always succeeded, despite numerous errors in the robots' movements. For instance, although the robots that we used for our first experiments (ALICE) were calibrated so as to turn by $90^{\circ}$ when detecting a wavefront, their real turning angle would vary from 0 to sometimes much over $90^{\circ}$. However, this did not obstruct the aggregation process and in all experiments we performed, the robots managed to gather in a tight area. 


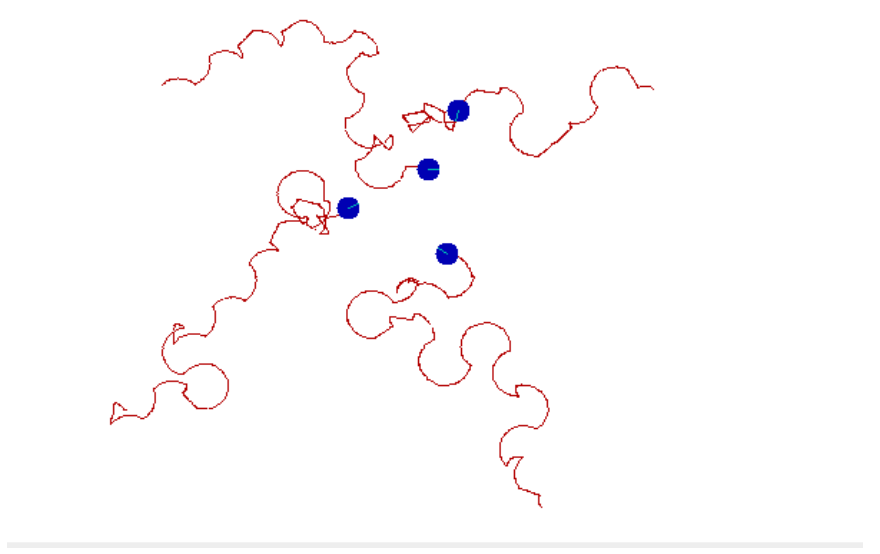

Figure 13: Recorded trajectories for the aggregation of 4 robots.

Figure 13 presents an example of an aggregation process with four robots. As it can be seen, the four trajectories are convergent but are also very noisy. From an observer point of view, the aggregation also looks slow : with our settings, the time needed to make the robots come in a close area typically varied from 1 to 5 minutes. The key point is that these drawbacks should not be interpreted as a default of our system: it is precisely the use of randomness in the triggering of the waves and in the movements of the robots that guarantees the system's properties of robustness. As observed from the simulations we carried out, it should also be understood that it is this "clever" use of randomness that will allow the system to scale and be applied to a large team or to a swarm of robots. Indeed, all happened as if the system "wants" to minimize the distance between robots and each time step. From an observer point of view, one sees that the "enveloppe" that contains all the robots progressively shrinks until robots get close to another.

The only case were the aggregation process was blocked was when spurious sources of emissions of waves existed. In our case, such errors resulted from "false" detections but one can also imagine that they come from robots that will always be emitting. We observed that these errors caused no problem when they were were rare, but in the case where a "ghost source" continuously triggered waves, the aggregation process was significantly perturbed. Indeed, the robots could not separate the consecutive waves which brought them to turn randomly. 


\section{Obstacle Avoidance with Kheperalll Robots}

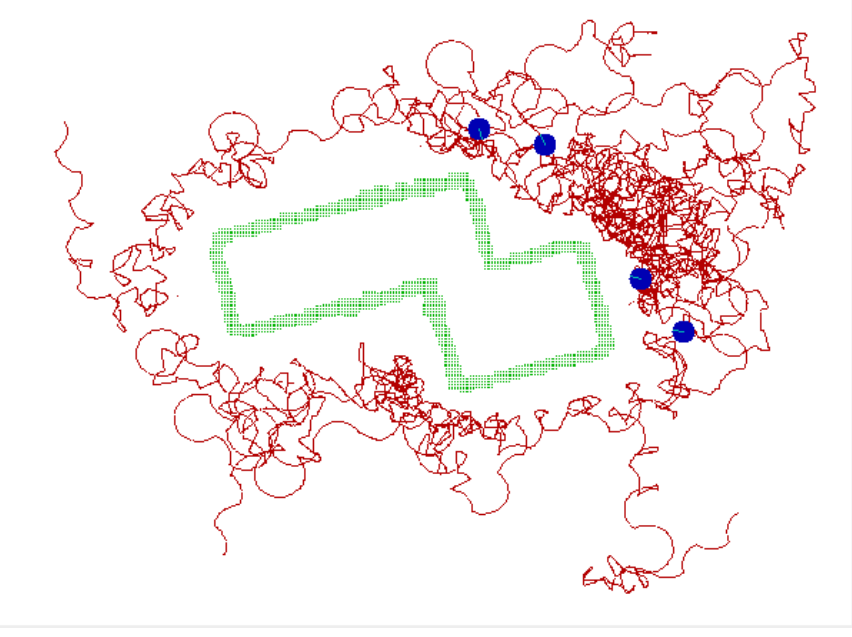

Figure 14: Recorded trajectories for the obstacle avoidance algorithm that uses multiple agitation moves (strategy 3)

Our next step was to consider environments with physical obstacles that we added to the environment. This experiments were made to study how the gathering process is affected by a change which, according to our assumptions, the agents are not aware of. From the perspective of the CA environment that propagates the reaction-diffusion waves, permanent obstacles were represented as cells with a fixed state.

From the robot's perspective, we used three strategies to cope with such obstacles. The common denominator of these strategies was that whenever an obstacle was present in the path of a robot, the robot would immediately stop moving, whatever its current state.

1) The first and simplest method was to add random moves to the agents. Such moves would occur when the agent was idle, that is, not performing a chemotaxis move or being in a refractory state. They consisted of a turn towards a random direction followed by moving for a fixed distance towards this direction. The use of this strategy increased the aggregation time, but performed good in the presence of both permanent and non-permanent obstacles.

2) The second strategy was to make a robot move backwards on a given distance when it meets an obstacle. We observed that this method has a good performance in the case where there are no permanent obstacles but that it created deadlocks in some configurations with permanent obstacles. Such deadlocks occur when the size of the obstacle is greater than the "backtracking" distance of the robot. Figure 15 illustrates a deadlock scenario. First, the robot detects a wave and turns towards the obstacle; it then backtracks and now faces the obstacle. When the next wave is detected, it turns left and approaches the obstacle. When the robot detects the obstacle, it backtracks and this time faces it left. The whole process repeats again and again, introducing a deadlock. The third strategy we used was a slight improvement on the backtracking method. 
Instead of "blindly" moving backwards, and since it was easy to determine which side of the robot is not blocked by the obstacle, the robot would move towards the "most free" area. Surprisingly, this method performed even worse than the simple backtracking and increased the deadlocks instead of resolving them. The reason for such a behavior was that the robots were always attracted in the same area and had less possibilities to escape the deadlock by pure chance.

3) The most promising strategy we employed was a variation on the first method which consisted of a combination of two random moves. First, the agitatory movement was preserved. Second, whenever an obstacle was blocking a robot and the robot wanted to move towards that direction it would perform a sequence of several agitation movements, increasing the chances to move towards a free area. This strategy resolved many deadlocks in the process and gave the best results in terms of a trade-off between minimizing the aggregation tie and keeping the individual behavior of the robots simple (see Fig. 14 for an example of trajectory).

There are of course many other possible methods for avoiding deadlocks. For instance, the robots follow the gradient of the reaction-diffusion waves instead of just detecting the wavefronts. However, as already mentioned, this would imply a more complex and possibly less robust behavior of the system.

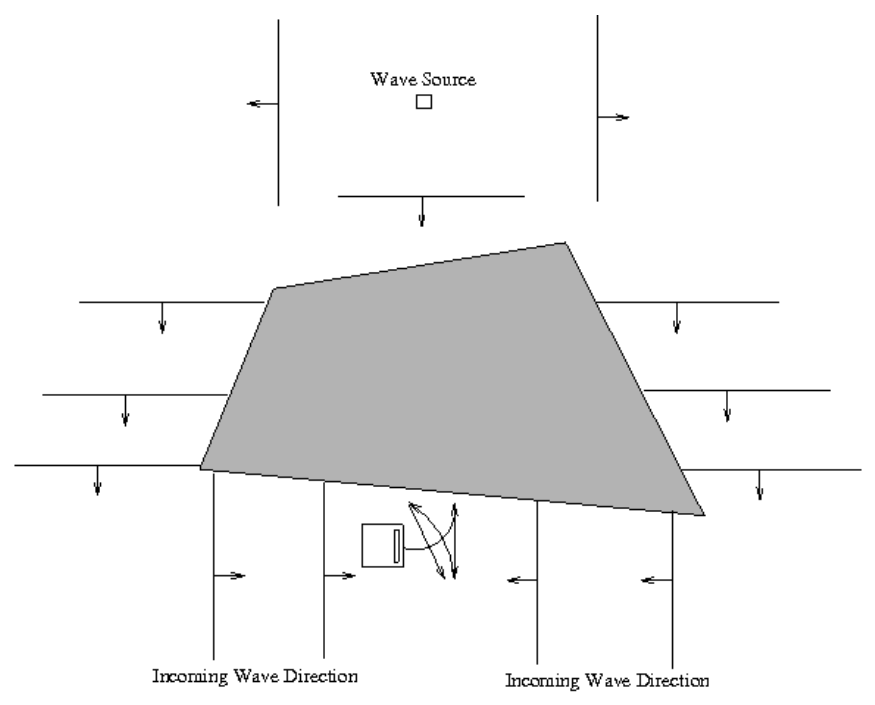

Figure 15: Example of deadlocks introduced by the "backtracking" obstacle avoidance method

In short, the two basic features of the amoebae aggregation scheme is that it works using only local operations and stigmergy, as well as the fact that it delegates most of the communication overhead to the environment. The operations are local both for the agents and for the CA-based environment. This significantly simplifies the agent design and agent logic, but also requires the existence of active cells to relay the messages. 


\section{CONCLUSIONS AND FUTURE WORK}

We presented our first results on the application of a decentralized aggregation scheme on two different types of robots. Our method to achieve the aggregation used a stigmergic principle and employed only local interactions of the robots with their environment. The experiments carried out allowed us to observe that the aggregation scheme is simple to implement and resists various perturbations on the robots. The main advantage of our method is to use only minimal assumptions on the robots abilities and on their sensors (number, precision, fidelity, etc.). The main difficulty for implementing the aggregation scheme is the design of an active environment to relay the messages without attenuation. In our experiments, the environment was simulated by an external (centralized) computation and projected on the robots. We also considered the case where this environment contained permanent obstacles and observed that, contrarily to the simplified discrete model, we need to add extra feature to the robots in order to solve some "deadlock" situations.

So far, our experiments involved no more than a few robots. Nevertheless, from the CA simulations we have observed that the model maintains good aggregation properties even for high number of agents (up to a few hundreds). These aggregation properties are maintained through the change of scale mainly because of the bio-inspired nature of the scheme and its "intelligent" use of randomness (no need for electing a "leader" is involved). In the case were a swarm of robots is used, the challenge would be to keep a "reasonable" aggregation time. This goal could be achieved by minimizing the number of colliding waves that annihilate. Indeed, not all the emitted waves reach all agents and therefore this somehow slows down the aggregation. From a previous research on the aggregation scheme (Vlassopoulos \& Fatès, 2010), we know that there is an optimal value of the firing rate. It is thus an open question to know how this optimal value depends on the number of robots and on other parameters.

As a part of our ongoing research, we are developing a model that simulates the KheperaIII ROMEA interaction. The model used allows us to test and verify our observations and try new algorithms for obstacle avoidance and deadlock prevention. In order to gain some realism, we intend to develop intermediate layers to run simulations on larger or more complex environments. Our goal is to investigate whether the good scaling properties observed with the discrete CA simulation model are kept with these more realistic models.

Finally, we would like to study how the system behavior varies as we change the assumptions made on the agents abilities. Indeed, increasing the "level of cooperation" of the agents with the environment could provide a more efficient gathering process, at least in the case where obstacles are present. This raises the question of how to set the trade-off between simplifications made in the agents and the robustness and efficiency of the aggregation process. 


\section{REFERENCES}

Garnier S. et al. (2008), The Embodiment of Cockroach Aggregation Behavior in a Group of MicroRobots, Artificial Life, 14, 387-408

Garnier S. et al. (2009), Self-organized aggregation triggers collective decision making in a group of cockroach like robots, Adaptive Behavior, 17(2), 109-133

Sugihara K. \& Suzuki I. (1996), Distributed Algorithms for Formation of Geometric Patterns with many Robots, Journal of Robotic Systems, 13(3), 127-139

Ando H., Oasa Y., Suzuki I. \& Yamashita M., (1999) Distributed memoryless point convergence algorithms for mobile robots with limited visibility, IEEE Transactions on Robotics and Automation, 15(5), 818-828

Doran J. E., Franklin S., Jennings N. R. \& Norman T. J., On Cooperation in Multi-Agent Systems, Knowledge Engineering Review, 12(3), 309-314, Cambridge University Press

Fatès, N., (2010) Solving the decentralised gathering problem with a reaction-diffusionchemotaxis scheme - Social amoebae as a source of inspiration, Swarm Intelligence, 4, 91-115, Springer New York

Spicher A., Fatès N. \& Simonin O., (2010) Translating Discrete Multi-Agents Systems into Cellular Automata: Application to Diffusion-Limited Aggregation, Proceedings of ICAART'09 Revised Selected Papers, Vol: 67, (pp 270-282), Springer Berlin

Greenberg J. M., Hassard B. D. \& Hastings S. P., (1978) Pattern formation and periodic structures in systems modeled by reaction-diffusion equations, Bulletin of the AMS, 84(6), 12961327, (http://projecteuclid.org/euclid.bams/1183541468)

Beaufort N., Charpillet F., Rochel O. \& Simonin O., Interactive table to study interactions between swarms of robots and active environments, (Tech. Rep.), France: LORIA - INRIA Nancy http://hal.inria.fr/inria-00575650

Vlassopoulos N. \& Fatès N., (2010) How fast can Virtual Amoebae aggregate? Analysis for the Optimal Firing Rate in an Instance of the Reaction-Diffusion-Chemotaxis Aggregation Scheme (Tech. Rep)., France: LORIA - INRIA Nancy, http://hal.archives-ouvertes.fr/inria-00462156/fr/

Prencipe G., Impossibility of Gathering by a set of Autonomous Mobile Robots, (2007) Theoretical Computer Science, 384(2-3), 222-231

Flocchini P. et al., (2005) Gathering of Asynchronous Robots with Limited Visibility, Theoretical Computer Science, 337(1-3), 147-168 